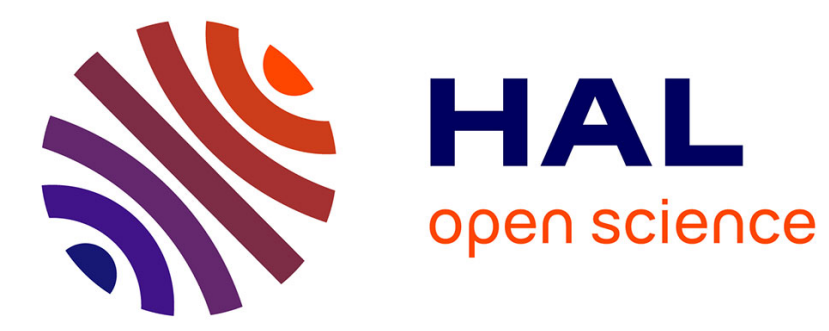

\title{
Surface wave interferometry by cross-correlation and deconvolution
}

\author{
Adnand Bitri, Gilles Grandjean, Kevin Samyn
}

\section{To cite this version:}

Adnand Bitri, Gilles Grandjean, Kevin Samyn. Surface wave interferometry by cross-correlation and deconvolution. First Break, 2011, 29 (5), 4 p. 10.3997/1365-2397.2011015 . hal-00567771

\section{HAL Id: hal-00567771 https://hal-brgm.archives-ouvertes.fr/hal-00567771}

Submitted on 21 Feb 2011

HAL is a multi-disciplinary open access archive for the deposit and dissemination of scientific research documents, whether they are published or not. The documents may come from teaching and research institutions in France or abroad, or from public or private research centers.
L'archive ouverte pluridisciplinaire HAL, est destinée au dépôt et à la diffusion de documents scientifiques de niveau recherche, publiés ou non, émanant des établissements d'enseignement et de recherche français ou étrangers, des laboratoires publics ou privés. 


\title{
Surface wave interferometry by cross-correlation and deconvolution
}

\author{
A. Bitri ${ }^{1, *}$, G. Grandjean ${ }^{1}$ and K. Samyn ${ }^{1}$ \\ ${ }^{1}$ BRGM/RNSC, 3 Avenue C. Guillemin, BP 6009 Orléans, Cedex 2, France. \\ *Corresponding author, E-mail: a.bitri@brgm.fr
}

\begin{abstract}
Frequency analysis of surface waves propagation is an efficient tool to retrieve the vertical shear-wave velocity profile. Surface waves result from the interaction of elastic body waves with a free surface. They are commonly generated by impulsive active sources but are also present in ambient noise. Here we compare the results of interferometric estimation of surface-wave dispersion from controlled-noise sources by using both cross-correlation and deconvolution methods. The crucial step in both methods lies in the dispersion image computation that conditions the reliability of the dispersion curve, and therefore the inversion results. We have found that the dispersion characteristics of surface waves are better defined when the deconvolution method is used. This better definition is probably due to the relative independence of the deconvolved output from the source function.
\end{abstract}

\section{Introduction}

Surface waves generated during seismic surveys can be an important source of information regarding the structure and properties of the shallow subsurface. Surface waves are dispersive, meaning that each frequency component of the wave travels at a different velocity, because at longer wavelengths the amplitude of the disturbance decays more slowly with depth.

Dispersion curves, plotted in the frequency-velocity domain, constitute the main input used in inversion schemes leading to estimations of the shear-wave (S-wave) velocity-depth profile. 
Different forms of dispersion analysis have been proposed for estimating S-wave velocitydepth profiles for a large variety of applications (e.g., Gabriels et al., 1987; Bitri et al., 1998; Grandjean and Bitri, 2006; Socco et al., 2009).

Seismic surface waves can be recorded through active source experiments operating multi-channel devices (Park et al., 1999). Recently, methods based on cross-correlation of ambient noise have been successfully tested for recovering surface wave dispersion from few hundred metres to several hundred kilometres (e.g., Campillo and Paul, 2003; Shapiro et al., 2005; Nunziata et al., 2009). The main advantages of such methods are the low cost of the data acquisition compared to active source surveys, and the fact that the sites where the data are generally acquired, e.g., industrial areas, city centres, roads or highways, are ideal for noise analysis methods. In addition, seismic surface wave signals commonly have higher amplitudes than other types of seismic waves, which may explain why they are so easy to analyse in the ambient noise.

Seismic interferometry refers to the study of interference phenomena between pairs of seismic signals in order to obtain information from their differences. These methods have found many applications in different fields of seismology (e.g., Campillo and Paul, 2003; Shapiro et al., 2005). There are many other examples of interferometry applications in other fields such as ultrasound (Malcolm et al., 2004) and helioseismology (Rickett and Claerbout, 1999). Curtis et al. (2006) and Larose at al. (2006) provide a comprehensive interdisciplinary review of seismic interferometry.

The main objective of seismic interferometry is to obtain the impulse response between receivers, without knowledge of model parameters (Lobkis and Weaver, 2001; Wapenaar et al., 2004). The main mathematical operation used to study interference is crosscorrelation of recorded data. Based on perturbation theory and representation theorems, 
Vasconcelos and Snider (2008a,b) show that interferometry can be performed by deconvolution for arbitrary media and multidimensional experiments.

In this article, we compare the results of interferometric estimation of surface-wave dispersion curves for controlled-noise sources using both cross-correlation and deconvolution methods. From our study, we show that the dispersion curves are better defined when the deconvolution method is used. This better definition is probably due to the relative independence of the deconvolved output from the source function.

\section{Interferometry by cross-correlation and deconvolution}

In this section, we summarize the cross-correlation and deconvolution operations. Following the work of Vasconcelos and Snider (2008a, 2008b), the cross-correlation of the wavefields measured at $r_{\mathrm{A}}$ and $r_{\mathrm{B}}$ gives

$$
C_{\mathrm{AB}}=|W(s, \omega)|^{2} G\left(r_{\mathrm{A}}, s, \omega\right) G^{*}\left(r_{\mathrm{B}}, s, \omega\right)
$$

where * denotes the complex conjugate, $G$ the Green's function and $W(s)$ the source power spectrum. In case of passive sources, we can integrate the cross-correlation operation over the surface $\Sigma$ that includes all the sources:

$$
\oint_{\Sigma} C_{\mathrm{AB}}=\left\langle|W(s, \omega)|^{2}\right\rangle G\left(r_{\mathrm{A}}, r_{\mathrm{B}}, \omega\right) G^{*}\left(r_{\mathrm{A}}, r_{\mathrm{B}}, \omega\right)
$$

where $\left\langle|W(s, \omega)|^{2}\right\rangle$ is the average power spectrum and $G\left(r_{\mathrm{A}}, r_{\mathrm{B}}, \omega\right)$ and $G^{*}\left(r_{\mathrm{A}}, r_{\mathrm{B}}, \omega\right)$ are the causal and acausal Green's functions for an excitation at $r_{\mathrm{A}}$ and a receiver at $r_{\mathrm{B}}$. The constructive interference of propagating signals along the surface $\Sigma \square$ from the sources to $r_{\mathrm{A}}$ and $r_{\mathrm{B}}$ is then sufficient to allow the construction of the Green's function between $r_{\mathrm{A}}$ and $r_{\mathrm{B}}$. The most direct contribution comes from the vicinity of $r_{\mathrm{A}}$ and $r_{\mathrm{B}}$ since the result is independent of the configuration of the surface $\Sigma$. 
On the other hand, the deconvolution of the wavefields measured at $r_{\mathrm{A}}$ and $r_{\mathrm{B}}$ is given

by

$D_{\mathrm{AB}}=\frac{G\left(r_{\mathrm{A}}, s, \omega\right) G^{*}\left(r_{\mathrm{B}}, s, \omega\right)}{\left|G\left(r_{\mathrm{B}}, s, \omega\right)\right|^{2}+\varepsilon^{2}}$

where $\varepsilon$ is a stabilization parameter. In contrast to Equation (2), when the two recorded signals are deconvolved the source function is cancelled, making the interferometric results independent of the source function. From Equation (3), integration over the surface $\Sigma$ including all the sources gives:

$\oint_{\Sigma} D_{\mathrm{AB}} d s=\oint_{\Sigma} \frac{G\left(r_{\mathrm{A}}, s, \omega\right) G^{*}\left(r_{\mathrm{B}}, s, \omega\right)}{\left|G\left(r_{\mathrm{B}}, s, \omega\right)\right|^{2}+\varepsilon^{2}} d s$

By expanding the denominator in a power series, Vasconcelos and Snider (2008a) showed that interferometry by deconvolution does not recover the acausal response between the two receivers. This is the main difference between interferometry and cross-correlation.

\section{Data acquisition and processing}

The dataset used in this study was acquired in a field adjacent to the BRGM site. The first experiment involved an active source (GISCO-ESS200 weight-drop source) and standard 10 $\mathrm{Hz}$ geophones placed at $5 \mathrm{~m}$ intervals along a straight line. A 24-channel Geometrics Geode seismograph was used for recording. The shot was located off-end from the geophone spread at $5 \mathrm{~m}$ from geophone 1 . The resulting shot gather (Figure 1a) shows a very clear surface wave of low frequency and a guided P-wave of higher frequency.

One of the key steps in the surface-wave analysis is to produce the dispersion curve in the frequency-velocity domain. The dispersion characteristics of surface waves are displayed by using a wavefield transformation from the time-offset $(t-x)$ domain into the phase velocityfrequency $(c-f)$ domain. Then shot gathers can be transformed into the intercept-slowness $(\tau$ - 
p) domain by using a slant-stacking method followed by a 1-D Fourier transform over the intercept time (McMechan and Yeldin, 1981; Moktar et al., 1988). Figure 1b shows the dispersion curve for the shot gather of Figure 1a. The fundamental mode of the surface wave (Rayleigh wave) and guided P-wave stand out clearly. The surface Rayleigh waves are sensitive to the S-wave velocity-depth profile and guided waves are sensitive to the $\mathrm{P}$-wave velocity-depth profile. Rayleigh and guided waves thus contain valuable information about physical parameters in a depth range that generally cannot be resolved by reflected or refracted waves. S-wave velocities can be estimated quickly from inversion of Rayleigh-wave dispersion, assuming a horizontally layered structure (Xia et al., 1999).

The second dataset was acquired using the same geophone spread. The system recorded 10 minutes of controlled noise (car moving along the seismic line), as in the experiment carried out by Holliday et al. (2008). The first step in processing was to split the data into many time windows. For each receiver pair, the corresponding time windows were first cross-correlated and then deconvolved. The results were integrated over time. The overlap time between windows was set at $3 \mathrm{~s}$ to provide sufficient delay time for the slowest surface wave to be analysed. Figure 2 a shows the results of cross-correlation for a virtual source located at receiver 1 . As predicted by the theory, the cross-correlation of the wavefield recorded by a given pair of receivers shows the filtered version of the causal and acausal Green's function sum. Figure $2 b$ shows the dispersion curve of the cross-correlated signals in Figure 2a. The fundamental and first higher-order mode can be identified on this figure, but with a low signal-to-noise ratio. Note that the first higher-order mode is not seen on the dispersion curve computed from the active shot gather (Figure 1b).

Figure 3 a shows the results of the deconvolution method for a virtual source located at receiver 1. Only acausal energy in the wavefield is highlighted by the deconvolution process. The dispersion curve (Figure $3 b$ ) shows the fundamental and the first higher-order modes with 
a high signal-to-noise ratio. Indeed, this method provides processed signals with a high energy content and a sufficient resolution to clearly distinguish between the different dispersive modes of the Rayleigh waves. This point is important because multimode inversion of surface waves reduces the ambiguity of inversion and improves the accuracy of S-wave velocity determination (O’Neill and Matsuoka, 2005).

\section{Conclusions}

We have presented a comparison between cross-correlation and deconvolution methods for estimating interferometric surface-wave signals with controlled-noise sources. The key step in the surface-wave analysis is the generation of reliable dispersion curves, since they are used to invert for S-wave velocity profiles. We have shown that the dispersion of surface waves is better defined when using the deconvolution method. The dispersion curve resulting from deconvolution shows high amplitude, not only in the fundamental propagation mode but also in the first higher-order mode, which is a great advantage compared to classical processing techniques.

\section{References}

Bitri, A., Le Bégat, S. and Baltassat, J.M. [1998] Shear-waves velocity determination of soils from in-situ Rayleigh wave measurements. Proceedings of $4^{\text {th }}$ EEGS Meeting, Extended Abstracts, 503-506.

Campillo, M. and Paul, A. [2003] Long-range correlations in the diffuse seismic coda. Science, 299, 547-549.

Curtis, A., Gerstoft, P., Sato, H., Snieder, R. and Wapenaar, K. [2006] Seismic interferometry - turning noise into signal. The Leading Edge, 25, 1082-1092. 
Gabriels, P., Sneider, R. and Nolet, G. [1987] In-situ measurements of shear wave velocity in sediments with higher mode Rayleigh waves. Geophysical Prospecting, 35, 187-196.

Grandjean, G. and Bitri, A. [2006] 2M-SASW: inversion of local Rayleigh wave dispersion in laterally heterogeneous subsurfaces: application to Super-Sauze landslide (France). Near Surface Geophysics, 4, 367-375.

Holliday, D. and Curtis, A. [2008] Seismic surface wave in suburban environment: active and passive interferometric methods. The Leading Edge, 27, 210-218.

Larose, E., Margerin, L., Derode, A., van Tiggelen, B., Campillo, M., Shapiro, N., Paul, A., Stehly, L. and Tanter, M. [2006] Correlation of random wavefields: an interdisciplinary review. Geophysics, 71, SI11-SI21.

Lobkis, O.I. and Weaver, R.L. [2001] On the emergence of the Green's function in the correlations of a diffuse field. Journal of the Acoustical Society of America, 110, 3011-3017. Malcolm, A., Scales, J. and van Tiggelen, B.A. [2004] Extracting the Greens function from diffuse, equipartitioned waves. Physical Review E, 70, 015601.

McMechan, G.A. and Yedlin, M.J. [1981] Analysis of dispersive waves by wave field transformation. Geophysics, 46, 869-874.

Moktar, T.A., Herrmann, R.B. and Russell, D.R. [1988] Seismic velocity and Q model for the shallow structure of the Arabian shield from short-period Rayleigh waves. Geophysics, 53, $1379-1387$.

Nunziata, C., De Nisco, G. and Panza, G.F. [2009] S-wave profiles from noise cross correlation at small scale. Engineering Geology, 105, 3-4.

O’Neill, A. and Matsuoka, T. [2005] Dominant higher surface-wave modes and possible inversion pitfalls. Journal of Environmental and Engineering Geophysics, 10, 185-201. Park, C.B., Miller, R.D. and Xia, J. [1999] Multi-channel analysis of surface waves. Geophysics, 64, 800-808. 
Rickett, J.E. and Claerbout, J.F. [1999] Acoustic daylight imaging via spectral factorization; helioseismology and reservoir monitoring. The Leading Edge, 19, 957-960.

Shapiro, N.M., Campillo, M., Stehly, L. and. Ritzwoller, M.H. [2005] High-resolution surface-wave tomography from ambient seismic noise. Science, 307, 1615-1618.

Socco, L.V., Boiero D., Foti, S. and Wisen, R. [2009] Laterally constrained inversion of ground roll from seismic reflection records. Geophysics, 74, G35-G45.

Vasconcelos, I. and Snieder, R. [2008a] Interferometry by deconvolution, Part 1-Theory for acoustic waves and numerical examples. Geophysics, 73, S115-S128.

Vasconcelos, I. and Snieder, R. [2008b] Interferometry by deconvolution: Part 2-Theory for elastic waves and application to drill-bit seismic imaging. Geophysics, 73, S129-S141.

Wapenaar, K., Thorbecke, J. and Dragonov, D. [2004] Relations between reflection and transmission responses of three-dimensional inhomogeneous media. Geophysical Journal International, 156, 179-194.

Xia, J., Miller, R.D. and Park, C.B. [1999] Estimation of near-surface shear-wave velocity by inversion of Rayleigh wave. Geophysics, 64, 691-700. 


\section{Figure captions}

Figure 1 (a) Field shot gather of 24 traces with high-frequency guided P-wave and lowfrequency surface wave. (b) Transform of the raw shot gather into the frequency-velocity domain. The fundamental surface wave mode and the dispersed guided P-wave stand out clearly.

Figure 2 (a) Causal and acausal surface waves obtained by cross-correlation of the controlled-noise source. The virtual source is at receiver 1. (b) Transform of the crosscorrelation results into the frequency-velocity domain. The dispersed fundamental mode and first higher-order mode stand out clearly.

Figure 3 (a) Causal surface waves obtained by deconvolution of the controlled-noise source. The virtual source is at receiver 1. (b) Transform of the deconvolution results into the frequency-velocity domain. The dispersed fundamental mode and first higher-order mode stand out clearly. 